\title{
Um método de controle robusto por rejeição ativa de distúrbios para sistemas MIMO incertos.
}

\author{
Josiel Alves Gouvêa* Alessandro Rosa Lopes Zachi ** \\ Luciano Santos Constantin Raptopoulos*** \\ * Faculdade de Engenharia de Controle e Automação, \\ CEFET-RJ/Campus Nova Iguaçu, RJ, (e-mail: \\ josiel.gouvea@cefet-rj.br). \\ ** Faculdade de Engenharia Eletrônica, CEFET-RJ, RJ (e-mail: \\ alessandro.zachi@cefet-rj.br) \\ *** Faculdade de Engenharia de Controle e Automação, \\ CEFET-RJ/Campus Nova Iguaçu, RJ, (e-mail: \\ luciano.raptopoulos@cefet-rj.br).
}

\begin{abstract}
This work approaches the problem of controlling uncertain Multiple-Input/MultipleOutput(MIMO) systems. An extension of the Active Disturbance Rejection Control (ADRC) is used, which consists in a structural change of the plant introducing filters in parallel with it. Using an Extended State Observer (ESO), the perturbation and unmodeled dynamics are estimated and used in a stabilizing controller that tracks a desired trajectory. A mathematical analysis and simulation results show the effectiveness of the proposed strategy.

Resumo: Este trabalho aborda o problema de controle de sistemas incertos com múltiplas entradas e saídas (MIMO). Utiliza-se uma extensão do Controlador por Rejeição Ativa de Distúrbios (ADRC), que consiste em uma mudança estrutural da planta introduzindo-se filtros em paralelo com mesma. Através de um Estimador de Ordem Estendida (ESO), a perturbação e a dinâmica não modelada são estimadas, incluindo a matriz de ganhos de controle, e utilizadas em um controlador estabilizante que rastreia uma trajetória desejada. Uma análise matemática e resultados de simulação mostram a eficácia da estratégia proposta.
\end{abstract}

Keywords: Uncertain systems; Robust control; Extended estimator; MIMO systems; Tracking trajectory.

Palavras-chaves: Controle robusto; Estimador estendido; Sistemas MIMO; Rastreamento de trajetória.

\section{INTRODUÇÃO}

Uma das maiores dificuldade no projeto dos sistemas de controle é a obtenção da descrição matemática do sistema, pois um erro nesta gera dinâmicas não modeladas ou incertezas nos valores dos parâmetros dinâmicos. Diz-se então que o sistema é incerto, sendo o seu controle um problema de grande relevância devido às suas aplicações industriais e acadêmicas. Dentre as diversas estratégias de controle para sistemas incertos propostas na literatura, relacionadas a plantas lineares e não-lineares, pode-se citar a estratégia Backsteping (Chen et al., 2015), Controle Adaptativo por Modelo de Referência (MRAC) (Selfridge and Tao, 2016), VS-MRAC (Hsu et al., 2014) e outros.

Além das incertezas do sistema, um outro problema fundamental no projeto de sistemas de controle é a rejeição eficiente de distúrbios externos, onde algumas interessantes e relevantes estratégias de controle não linear foram propostas ( $\mathrm{Li}$ et al., 2016; Mobayen and Tchier, 2017). Neste contexto, emergiu a estratégia de Controle por Rejeição Ativa de Distúrbios (ADRC) (Xue et al.,
2015), que consiste na utilização de um Estimador de Ordem Estendida (ESO) para estimar os sinais não mesuráveis da planta, incluindo as dinâmicas não modeladas e distúrbios externos, e usar estas estimativas em uma lei de controle estabilizante. As principais características do método ADRC são a implementação simples e a boa propriedade de robustez aos distúrbios externos, dinâmicas não modeladas e incertezas paramétricas. Devido a essas vantagens, extensões deste método foram desenvolvidas, possibilitando a sua aplicação em outras classes de sistemas. Como exemplo, em (Zheng et al., 2009) a estratégia ADRC foi estendida para sistemas com Múltiplas Entradas e Múltiplas Saídas (MIMO), sendo aplicada em um processo químico industrial para demonstrar o seu desempenho. Para sistemas de ordem mais alta, em (Zhang et al., 2016) é proposto o uso de observadores de ordem reduzida em cascata, onde os parâmetros de controle e estimação são mantidos para cada observador, sendo as estimativas dos valores paramétricos obtidas utilizando um procedimento de otimização de enxame de partículas de acordo com uma função custo estabelecida. Em (Sun et al., 2016) é proposta uma extensão do ADRC para tratar com 
sistemas de fase não mínima, onde é utilizado um controle feedfoward assistido por modelo para assegurar um erro de estado estacionário nulo com um mínimo tempo de acomodação. Contudo, como pode ser observado nesses e em outros trabalhos recentes envolvendo o ADRC (Guo et al., 2017; Xia et al., 2018), embora considere-se os parâmetros dinâmicos incertos, o ganho de controle é considerado conhecido. Portanto, o ADRC é pouco robusto às variações no ganho de controle. No entanto, em muitas aplicações práticas, tais como transporte de carga por robôs móveis e/ou drones, o valor da massa da carga e do centro de gravidade do sistema variam durante a execução da tarefa, o que provoca uma incerteza na matriz de inércia. Como esta matriz influencia fortemente no ganho de controle (Fossen, 1994; Popp and Schiehlen, 2010), o mesmo também tornase incerto.

Visando resolver o problema da incerteza no ganho de controle, em (Zachi et al., 2019) é proposta uma extensão do ADRC, denominada ADRC modificado. A modificação consiste em fazer uma alteração estrutural na planta, acrescentando-se um filtro em paralelo com a mesma. Um ESO é então projetado para a planta modificada com o objetivo de se obter uma estimativa que engloba o distúrbio externo, a dinâmica não modelada e as incertezas paramétricas, incluindo o ganho de controle, onde é somente necessário o conhecimento do sinal do mesmo. No entanto, como só foi analisado matematicamente o caso de sistemas com uma única entrada e saída (sistemas SISO), o ganho de controle é escalar. Para sistemas MIMO, a análise matemática de estabilidade de Zachi et al. (2019) só se aplicaria se o mesmo tivesse entradas desacopladas, pois, neste caso bem específico, um dado sistema MIMO pode ser representado como um conjunto de sistemas SISO.

Neste trabalho, o ADRC modificado é estendido para sistemas MIMO considerando também o acoplamento dos sinais de entrada, onde o ganho de controle passa a ser uma matriz de entrada. O controlador obtido possui todas as propriedades de robustez do ADRC padrão, com a vantagem de também ser robusto à variação da matriz de entrada, sendo o conhecimento do sinal dos componentes da diagonal principal da mesma a única exigência para a aplicação do controlador.

Este trabalho está organizado da seguinte forma: na Seção 2 é feita uma pequena apresentação do ESO e do ADRC básico para sistemas MIMO. O ADRC modificado é abordado na Seção 3, onde é feita uma análise matemática de estabilidade do controlador. Resultados de simulação são mostrados na Seção 4 utilizando um exemplo acadêmico. Finalmente, na Seção 5 são apresentadas as conclusões finais e propostas de trabalhos futuros.

\section{CONTROLADOR ADRC BÁSICO PARA SISTEMAS MIMO}

Considere um sistema MIMO descrito por

$$
\ddot{X}=F(X, W)+B \tau
$$

onde $X^{T}=\left[x_{1}, x_{2}, x_{3}\right]$ é o vetor de saídas, $W^{T}=$ $\left[w_{1}, w_{2}, w_{3}\right]$ é um vetor de distúrbios externos, $F^{T}(X, W)=$ $\left[f_{1}(X, \dot{X}, W), f_{2}(X, \dot{X}, W), f_{3}(X, \dot{X}, W)\right]$, sendo $f_{i}(X, W)$ funções não lineares desconhecidas, denominadas distúrbios generalizados, $B \in \mathbb{R}^{3 \times 3}$ é uma matriz, denominada matriz de ganhos de controle, com componentes $b_{i j}$ considerados, inicialmente, conhecidos e $\tau^{T}=\left[\tau_{1}, \tau_{2}, \tau_{3}\right]$ é o vetor de entrada. Por simplicidade, o vetor de distúrbios generalizados $F(X, W)$ será, doravante, representado por $F(t)$. Sabe-se então que, caso $F(t)$ fosse conhecido e considerando $B$ não singular, poderia ser proposta a lei de controle de rastreamento

$$
\tau=B^{-1}\left[-K_{1} E-K_{2} \dot{E}-F(t)+\ddot{X}^{*}\right]
$$

onde $E=X-X^{*}, X^{*}=\left[x_{1}^{*}, x_{2}^{*}, x_{3}^{*}\right]$ é um vetor de trajetórias de referência e $K_{1}, K_{2} \in \mathbb{R}^{3 \times 3}$ matrizes de ganho de controle definidas positivas. Contudo, em muitas aplicações, o vetor de distúrbios generalizados $F(t)$ pode envolver parâmetros dinâmicos incertos e/ou não modelados, além de representar uma combinação de distúrbios externos não mensuráveis. Em tais casos, a lei de controle (2) não pode ser calculada diretamente. Visando superar esse problema, é projetado um Estimador de Ordem Estendida (ESO) para estimar $F(\underline{t})$. O procedimento básico é definir um vetor de estados $\bar{X}$ para (1), mas incluindo $F(t)$ como uma variável de estado adicional, ou seja,

$$
\bar{X}(t)=\left[\begin{array}{c}
\bar{X}_{1} \\
\bar{X}_{2} \\
\bar{X}_{3}
\end{array}\right]=\left[\begin{array}{c}
X \\
\dot{X} \\
F(t)
\end{array}\right] .
$$

Assumindo que $F(t)$ é diferenciável, a representação do sistema de ordem estendida no espaço de estados é descrita por

$$
\begin{aligned}
& \dot{\bar{X}}=\underbrace{\left[\begin{array}{lll}
\mathbf{0} & I & \mathbf{0} \\
\mathbf{0} & \mathbf{0} & I \\
\mathbf{0} & \mathbf{0} & \mathbf{0}
\end{array}\right]}_{A} \bar{X}+\underbrace{\left[\begin{array}{l}
\mathbf{0} \\
I \\
\mathbf{0}
\end{array}\right]}_{\bar{B}} B \tau+\underbrace{\left[\begin{array}{l}
\mathbf{0} \\
\mathbf{0} \\
I
\end{array}\right]}_{B_{1}} \dot{F}(t), \\
& Y(t)=\underbrace{\left[\begin{array}{lll}
\mathbf{I} & \mathbf{0} & \mathbf{0}
\end{array}\right]}_{C} \bar{X},
\end{aligned}
$$

onde $\mathbf{0}$ é uma matriz de zeros e $I$ uma matriz identidade, ambas de dimensão 3 . Note que o par $(A, C)$ é observável. Portanto, a ideia é implementar o seguinte observador de ordem estendida (ESO) para estimar $\bar{X}$ :

$$
\dot{\hat{\bar{X}}}=(A-L C) \hat{\bar{X}}+B \tau+L Y
$$

onde $\hat{\bar{X}}^{T}=\left[\hat{\bar{X}}_{1}^{T}, \hat{\bar{X}}_{2}^{T}, \hat{\bar{X}}_{3}^{T}\right]$ é a estimativa de $\bar{X}$ e $L \in \mathbb{R}^{9 \times 3}$ é o ganho do estimador. Note, usando (4) e (5), que a dinâmica do erro de estimação $\tilde{\bar{X}}=\bar{X}-\hat{\bar{X}}$ é descrita por

$$
\dot{\tilde{\bar{X}}}=(A-L C) \tilde{\bar{X}}+B_{1} \dot{F}(t)
$$

Note também que, como a representação (4) é observável, os autovalores de $A-L C$ sempre poderão ser alocados livremente no Semiplano Lateral Esquerdo (SLE). Portanto, o sistema (6) é BIBO estável e, consequentemente, o erro de estimação $\tilde{\bar{X}}$ será limitado se $\dot{F}$ também for e convergirá exponencialmente para zero se $F(t)$ convergir para um valor constante. Assim, usando os estados estimados $\hat{\bar{X}}$ em (2), pode-se propor a lei de controle

$$
\tau=B^{-1}\left[-K_{1}\left(\hat{\bar{X}}_{1}-X^{*}\right)-K_{2}\left(\hat{\bar{X}}_{2}-\dot{X}^{*}\right)-\hat{\bar{X}}_{3}+\ddot{X}^{*}\right] .
$$

\subsection{Análise de estabilidade em malha fechada}

Definindo o vetor de trajetórias de referência como $\bar{X}^{*}=$ $\left[X^{*}, \dot{X}^{*}, 0\right]$ e o erro de rastreamento como $\bar{E}=\bar{X}-\bar{X}^{*}$, 
pode-se concluir, a partir de (4) e (7), que a dinâmica do erro de rastreamento e a lei de controle $\tau$ podem ser descritas por

$$
\begin{gathered}
\dot{\bar{E}}=A \bar{E}+\bar{B} B \tau+A \bar{X}^{*}+B_{1} \dot{F}-\dot{\bar{X}}^{*} \\
\tau=B^{-1}\left(-K \bar{E}+K \tilde{\bar{X}}+\ddot{X}^{*}\right)
\end{gathered}
$$

onde $K=\left[K_{1}, K_{2}, I\right]$. Substituindo (9) em (8), conclui-se que

$$
\dot{\bar{E}}=(A-\bar{B} K) \bar{E}+\bar{B} K \tilde{\bar{X}}+B_{1} \dot{F}+\overbrace{\bar{B} \ddot{X}^{*}+A \bar{X}^{*}-\dot{\bar{X}}^{*}}^{=0} .
$$

Portanto, utilizando (6) e (10), conclui-se que a representação de estados do sistema em malha fechada, com o ESO, é descrita por

$$
\left[\begin{array}{c}
\dot{\tilde{X}} \\
\dot{\bar{E}}
\end{array}\right]=\left[\begin{array}{cc}
A-L C & \mathbf{0} \\
\bar{B} K & A-\bar{B} K
\end{array}\right]\left[\begin{array}{c}
\tilde{\bar{X}} \\
\bar{E}
\end{array}\right]+\left[\begin{array}{c}
B_{1} \\
B_{1}
\end{array}\right] \dot{F}(t) .
$$

Portanto, como os polos do sistema em malha fechada são a união dos autovalores das matrizes $A-L C$ e $A-\bar{B} K$, os mesmos sempre podem ser alocados no semiplano lateral esquerdo, por uma escolha apropriada dos ganhos do ESO $L$ e do controlador $K$. Portanto, assumindo $\dot{F}$ limitado, o que é viável na prática, conclui-se que:

- o sistema em malha fechada é BIBO estável;

- o erro de rastreamento $\bar{E}$ é limitado;

- $\bar{E} \rightarrow 0$ se $\dot{F} \rightarrow 0$.

Note que o conhecimento da matriz de ganhos de controle $B$ é essencial para a implementação da estratégia de controle. Portanto, o ADRC básico é pouco robusto à variação de $B$, o que reduz a robustez da estratégia com relação à variação dos parâmetros dinâmicos. Esse problema é abordado pelo ADRC modificado, como pode ser visto na próxima seção.

\section{ADRC MODIFICADO}

Considere a classe de sistemas descrito por (1). Para fins de análise, a parte linear de $F(t)$ é separada de forma a aparecer explicitamente na representação da planta, como mostrado abaixo:

$$
\ddot{X}=\underbrace{-A_{1} \dot{X}-A_{0} X+G(t)}_{F(t)}+B \tau,
$$

onde $A_{1}, A_{2} \in \mathbb{R}^{3 \times 3}$ são matrizes constantes e $G(t) \in$ $\mathbb{R}^{3}$ é um distúrbio que engloba a dinâmica não linear e as perturbações externas. Antes de continuar com o desenvolvimento do ADRC modificado, são consideradas as seguintes hipóteses:

Hipótese 1. A norma do distúrbio $G(t)$ é limitada com derivada de primeira ordem também limitada, ou seja, $\|G(t)\|<G_{1},\|\dot{G}(t)\|<G_{2}$, sendo $G_{1}$ e $G_{2}$ limitantes conhecidos.

Hipótese 2. As matrizes de parâmetros da planta $A_{1} \mathrm{e}$ $A_{0}$ e a matriz de entrada $B$ são incertas, mas os limites superiores dos componentes de $A_{1}$ e $A_{0}$ e inferiores de $B$ são conhecidos e $B$ é não singular. Isso implica em

$$
\left\|A_{1}\right\|_{\infty}<M_{1} \quad\left\|A_{0}\right\|_{\infty}<M_{0} \quad M_{b}<\|B\|_{\infty}
$$

onde $M_{1}, M_{0}$ e $M_{b}$ são constantes conhecidas. Além disso, os sinais dos componentes da diagonal principal de $B$ são conhecidos.
A Hipótese 1 é fundamental para a escolha da banda passante do estimador de estados. Quanto maior for $\dot{G}(t)$, maior deverá ser a banda passante do estimador. Já a Hipótese 2 permite considerar uma classe mais geral de plantas que possuem um conjunto completo de parâmetros incertos.

\subsection{Metodologia proposta}

A ideia do ADRC modificado é implementar uma mudança estrutural na planta (1), considerando os sinais da entrada e da saída da mesma, visando a obtenção de um novo sistema dinâmico com características que facilitam o projeto do ADRC. Para isso, é introduzida uma matriz de ganhos constantes $\beta \in \mathbb{R}^{3 \times 3}$ em série com a planta e uma matriz diagonal de filtros de segunda ordem

$$
D(s)=(s I+\Lambda)^{-2} I s
$$

em paralelo com a mesma, como mostra a Figura 1, onde $\Lambda=\gamma I$ e $\gamma \in \mathbb{R}$ é uma constante positiva tal que $(s+$ $\gamma)^{2}=s^{2}+\alpha_{1} s+\alpha_{0}$ seja um polinômio estável. Portanto,

$$
D(s)=\left[\begin{array}{ccc}
D_{1}(s) & 0 & 0 \\
0 & D_{2}(s) & 0 \\
0 & 0 & D_{3}(s)
\end{array}\right]
$$

onde

$$
D_{i}(s)=\frac{s}{(s+\gamma)^{2}} .
$$

Baseado na configuração da Figura 1, conclui-se que:

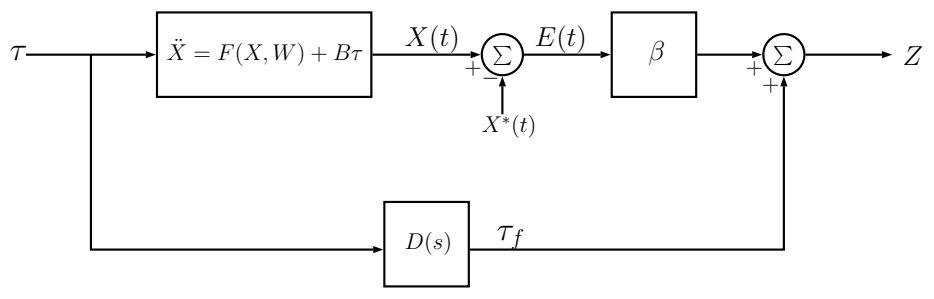

Figura 1. Diagrama de bloco da planta modificada.

$$
\begin{aligned}
Z(t) & =\beta E(t)+\tau_{f}(t), \\
E(t) & =X(t)-X^{*}(t), \\
\ddot{\tau}_{f} & =-\Lambda_{1} \dot{\tau}_{f}-\Lambda_{0} \tau_{f}+\dot{\tau},
\end{aligned}
$$

onde $\Lambda_{1}=\alpha_{1} I$ e $\Lambda_{0}=\alpha_{0} I$. Note, a partir de (13), que $\Lambda_{1}=2 \Lambda$ e $\Lambda_{0}=\Lambda^{2}$. Diferenciando (16) duas vezes em relação ao tempo e utilizando (12) e (17), obtém-se

$$
\ddot{Z}=\beta\left(-A_{1} \dot{X}-A_{0} X+G(t)+B \tau-\ddot{X}^{*}\right)+\ddot{\tau}_{f} .
$$

Como $\tau_{f}=Z-\beta E$, então pode concluir a partir de (18) que

$$
\ddot{\tau}_{f}=-\Lambda_{1}(\dot{Z}-\beta \dot{E})-\Lambda_{0}(Z-\beta E)+\dot{\tau} .
$$

Então, substituindo (20) em (19), chega-se na equação dinâmica da planta modificada:

$$
\ddot{Z}+\Lambda_{1} \dot{Z}+\Lambda_{0} Z=\Omega(t)+\dot{\tau}
$$

onde o novo distúrbio generalizado $\Omega(t)$ é descrito por

$\Omega(t)=\beta\left(-A_{1} \dot{X}-A_{0} X+G(t)+B \tau-\ddot{X}^{*}+\Lambda_{1} \dot{E}+\Lambda_{0} E\right)$.

Note que, em (21), a matriz de entrada $B$ é inserida no novo distúrbio generalizado $\Omega(t)$ e o novo sinal de entrada do sistema é $\dot{\tau}$, que possui $I$ como matriz de entrada. Portanto, se $\Omega(t)$ fosse conhecido, poderia ser proposta 
a lei de controle de rastreamento de trajetória $\dot{\tau}=-\Omega(t)$. No entanto, como o novo distúrbio generalizado $\Omega(t)$ é incerto, é novamente utilizado o ESO para estimá-lo. Assim, definindo-se as variáveis de estado $\bar{Z}_{1}=Z, \bar{Z}_{2}=\dot{Z}$ e $\bar{Z}_{3}=\Omega(t)$, a representação de (21) no espaço de estados é descrita por

$$
\begin{aligned}
& {\left[\begin{array}{c}
\dot{\bar{Z}}_{1} \\
\dot{\bar{Z}}_{2} \\
\dot{\bar{Z}}_{3}
\end{array}\right]=\underbrace{\left[\begin{array}{ccc}
\mathbf{0} & I & \mathbf{0} \\
-\Lambda_{0} & -\Lambda_{1} & I \\
\mathbf{0} & \mathbf{0} & \mathbf{0}
\end{array}\right]}_{\bar{A}} \underbrace{\left[\begin{array}{c}
\bar{Z}_{1} \\
\bar{Z}_{2} \\
\bar{Z}_{3}
\end{array}\right]}_{\bar{Z}}+\underbrace{\left[\begin{array}{c}
\mathbf{0} \\
I \\
\mathbf{0}
\end{array}\right]}_{\bar{B}} \dot{\tau}+\underbrace{\left[\begin{array}{c}
\mathbf{0} \\
\mathbf{0} \\
I
\end{array}\right]}_{\Gamma} \dot{\Omega}(t)} \\
& Z=\underbrace{\left[\begin{array}{lll}
I \text { 0 } & \mathbf{0}
\end{array}\right]}_{\bar{C}} \bar{Z} .
\end{aligned}
$$

Para facilitar o projeto do estimador, é utilizada a matriz transformação de estados

$$
\bar{Z}=\underbrace{\left[\begin{array}{ccc}
I & \mathbf{0} & \mathbf{0} \\
-\Lambda_{1} & I & \mathbf{0} \\
\mathbf{0} & \mathbf{0} & I
\end{array}\right]}_{T} \bar{Z}_{o}
$$

onde $\bar{Z}_{o}^{T}=\left[\begin{array}{lll}\bar{Z}_{o 1}^{T} & \bar{Z}_{o 2}^{T} & \bar{Z}_{o 3}^{T}\end{array}\right]$ é o vetor de estados na forma canônica observável. Note que $Z=\bar{Z}_{1}=\bar{Z}_{o 1}$ e $\Omega(t)=$ $\bar{Z}_{3}=\bar{Z}_{o 3}$. Sabe-se então que

$$
\begin{aligned}
& \dot{\bar{Z}}_{o}=\underbrace{\left[\begin{array}{ccc}
-\Lambda_{1} & I & \mathbf{0} \\
-\Lambda_{0} & \mathbf{0} & I \\
\mathbf{0} & \mathbf{0} & \mathbf{0}
\end{array}\right]}_{\bar{A}_{o}} \bar{Z}_{o}+\underbrace{\left[\begin{array}{l}
\mathbf{0} \\
I \\
\mathbf{0}
\end{array}\right]}_{\bar{B}_{o}} \dot{\tau}+\underbrace{\left[\begin{array}{l}
\mathbf{0} \\
\mathbf{0} \\
I
\end{array}\right]}_{\Gamma_{o}} \dot{\Omega}(t) \\
& Z=\underbrace{\left[\begin{array}{lll}
I & \mathbf{0} & \mathbf{0}
\end{array}\right]}_{\bar{C}_{o}} \bar{Z}_{o} .
\end{aligned}
$$

na qual $\bar{A}_{o}=T^{-1} \bar{A} T, \bar{B}_{o}=T^{-1} \bar{B}, \Gamma_{o}=T^{-1} \Gamma$ e $\bar{C}_{o}=$ $\bar{C} T$. Portanto, a equação do ESO para a representação observável (25) é

$$
\dot{\bar{Z}}_{o}=\left(\bar{A}_{o}-\bar{L}_{C_{o}}\right) \hat{\bar{Z}}_{o}+\bar{B}_{o} \dot{\tau}+\bar{L} Z
$$

onde $\hat{\bar{Z}}_{o}$ é a estimativa de $\bar{Z}_{o}, \bar{L}^{T}=\left[\begin{array}{lll}\bar{L}_{1}^{T} & \bar{L}_{2}^{T} & \bar{L}_{3}^{T}\end{array}\right]$ é o vetor de ganhos definido por

$$
\operatorname{det}\left[s I-\left(\bar{A}_{o}-\bar{L} \bar{C}_{o}\right)\right]=\left(s I+w_{0} I\right)^{3}
$$

e $\omega_{0} \in \mathbb{R}$ é uma constante positiva. Pode-se concluir então que

$$
\bar{L}_{1}=3 \omega_{0} I-\Lambda_{1}, \bar{L}_{2}=3 \omega_{0}^{2} I-\Lambda_{0}, \bar{L}_{3}=3 \omega_{0}^{3} .
$$

Portanto, a lei de controle para a planta modificada (21) pode agora ser descrita em termos de $\hat{\bar{Z}}_{o 3}$, a estimativa de $\Omega(t)$ :

$$
\dot{\tau}=-\hat{\bar{Z}}_{o 3} \text {. }
$$

Note que a análise da convergência de $\hat{\bar{Z}}_{\text {o3 }}$ para o distúrbio generalizado $\Omega(t)$ é essencial para verificar a estabilidade do sistema em malha fechada. Essa análise é implementada na próxima seção.

\subsection{Análise de convergência do ESO}

A partir de (25) e (26), pode-se concluir que a equação do erro de estimação é descrita por

$$
\begin{aligned}
& \dot{\overline{\bar{Z}}}=\left(\bar{A}_{o}-\bar{L} \bar{C}_{o}\right) \tilde{\bar{Z}}+\Gamma_{0} \dot{\Omega} \\
& E_{\Omega}=\Omega(t)-\hat{\bar{Z}}_{o 3}=\left[\begin{array}{lll}
0 & 0 & I
\end{array}\right] \tilde{\bar{Z}}
\end{aligned}
$$

onde $\tilde{\bar{Z}}=\bar{Z}_{o}-\hat{\bar{Z}}_{o}$.

Para escrever a equação do erro de estimação no domínio da frequência, é conveniente expressar (30) na forma controlável. Então, considerando a transformação

$$
\tilde{\bar{Z}}=\underbrace{\left[\begin{array}{ccc}
\mathbf{0} & \mathbf{0} & \mathbf{I} \\
\mathbf{0} & I & \Lambda_{1}+\bar{L}_{1} \\
\mathbf{0} & \Lambda_{1}+\bar{L}_{1} & \Lambda_{0}+\bar{L}_{2}
\end{array}\right]}_{T_{c}} \tilde{\bar{Z}}_{c},
$$

onde $\tilde{\bar{Z}}_{c}^{T}=\left[\begin{array}{ccc}\tilde{\bar{Z}}_{c_{1}}^{T} & \tilde{\bar{Z}}_{c_{2}}^{T} & \tilde{\bar{Z}}_{c_{3}}^{T}\end{array}\right]$, a representação de (30) na forma controlável é

$$
\begin{aligned}
\dot{\tilde{Z}}_{c} & =\left[\begin{array}{ccc}
-\Lambda_{1}+\bar{L}_{1} & -\Lambda_{0}+\bar{L}_{2} & -L_{3} \\
I & \mathbf{0} & \mathbf{0} \\
\mathbf{0} & I & \mathbf{0}
\end{array}\right] \tilde{\bar{Z}}_{c}+\left[\begin{array}{l}
I \\
\mathbf{0} \\
\mathbf{0}
\end{array}\right] \dot{\Omega} \\
E_{\Omega} & =\left[\begin{array}{lll}
I & \bar{L}_{1}+\Lambda_{1} & \bar{L}_{2}+\Lambda_{0}
\end{array}\right] \tilde{\bar{Z}}_{c} .
\end{aligned}
$$

Portanto, no domínio da frequência, conclui-se, usando (28), que

$$
\begin{aligned}
E_{\Omega}= & {\left[s^{3} I+s^{2} 3 \omega_{0} I+s 3 \omega_{0}^{2} I\right]\left[s^{3} I+\right.} \\
& \left.+s^{2} 3 \omega_{0} I+s 3 \omega_{0}^{2} I+\omega_{0}^{3} I\right]^{-1} \Omega .
\end{aligned}
$$

Após uma pequena manipulação algébrica, conclui-se que

$$
E_{\Omega}(t)=[I-\omega_{0}^{3} \underbrace{\left(s^{3} I+3 s^{2} \omega_{0} I+3 s \omega_{0}^{2} I+\omega_{0}^{3} I\right)^{-1}}_{\left(s I+\omega_{0} I\right)^{-3}}] \Omega(t) .
$$

Dado que, como se observa em $(30), E_{\Omega}=\Omega-\hat{\bar{Z}}_{o 3}$, então

$$
\hat{\bar{Z}}_{o 3}(t)=\underbrace{\left[\frac{\omega_{0}^{3}}{\left(s+\omega_{0}\right)^{3}}\right]}_{H(s)} \Omega(t),
$$

na qual a função de transferência $H(s)$ é aplicada nos três elementos do vetor de distúrbios generalizados $\Omega(t)$.

Observação 1. É importante mencionar que, a adoção de uma simultânea representação nos domínios do tempo e da frequência em (34) e (35) é somente para fins de análise. No caso de $\Omega(t)$ envolver uma função não linear dos estados da planta, a definição $\Omega(s)$ no domínio da frequência pode não ser consistente. Assim, como deseja-se analisar a amplitude do sinal do erro de estimação em (34) sem perder a generalidade, acreditamos que, neste trabalho, este é um formalismo matemático mais apropriado para representar a relação entrada/saída.

Note, a partir de (35), que $H(s) \in$ ] 0;1 [. Além disso, se $\omega_{0}$ for escolhido muito maior que a maior frequência de $\Omega(t)$, então, neste caso, teríamos $\hat{\bar{Z}}_{o 3}(t)=\Omega(t)$.

\subsection{Análise de estabilidade e rastreamento em malha fechada.}

A partir de (35) e das conclusões das seção anterior, sabese que pode ser definida uma variável $\left.c_{0} \in\right] 0 ; 1[$ de forma a escrever a lei de controle (29) como

$$
\dot{\tau}=-c_{0} \Omega(t) \text {. }
$$

Substituindo (22) em (36), tem-se

$\dot{\tau}=-c_{0} \beta\left(-A_{1} \dot{X}-A_{0} X+G(t)+B \tau-\ddot{X}^{*}+\Lambda_{1} \dot{E}+\Lambda_{0} E\right)$ 
Portanto, utilizando (12), conclui-se que

$$
\dot{\tau}=-c_{0} \beta\left(\ddot{E}+\Lambda_{1} \dot{E}+\Lambda_{0} E\right) .
$$

Lembrando que $\Lambda_{1}=2 \Lambda$ e $\Lambda_{0}=\Lambda^{2}$, a lei de controle (38) pode ser descrita, no domínio da frequência, por

$$
\tau=\left[-\frac{1}{s} c_{0} \beta(s I+\Lambda)^{2}\right] E \text {. }
$$

Observe, de (39) e (12), que o sistema em malha fechada pode ser representado pela Figura 2. Reorganizando os

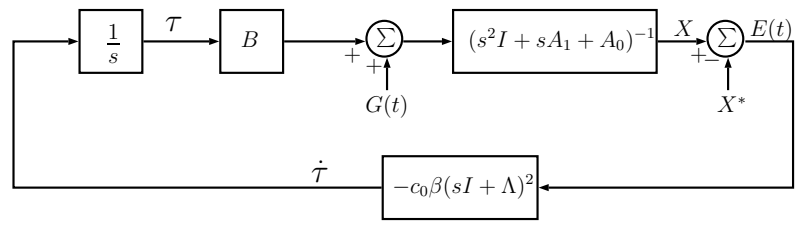

Figura 2. Diagrama de bloco do sistema em malha fechada.

blocos da Figura 2 para colocar o sinal $X(t)$ como saída e os sinais $G(t)$ e $X^{*}(t)$ como entradas, chega-se ao diagrama de blocos da Figura 3, onde

$P(s)=s^{3} I+\left(A_{1}+c_{0} B \beta\right) s^{2}+\left(A_{0}+\Lambda_{1} c_{0} B \beta\right) s+c_{0} B \beta \Lambda_{0}$.

Considerando a Hipótese 2 , pode-se escolher $c_{0}, \beta, \Lambda_{1} \mathrm{e}$

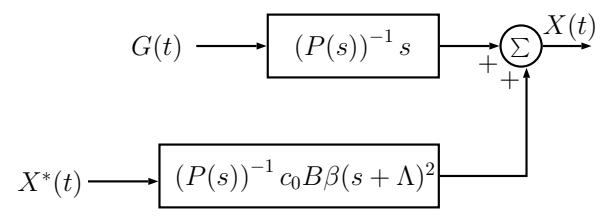

Figura 3. Diagrama de bloco do sistema em malha fechada.

$\Lambda_{2}$ tal que $\left\|c_{0} B \beta\right\|_{\infty} \gg M_{1}$ e $\left\|\Lambda_{1} c_{0} B \beta\right\|_{\infty} \gg M_{0}$. Neste caso,

$$
P(s) \approx s^{3} I+c_{0} B \beta s^{2}+\Lambda_{1} c_{0} B \beta s+c_{0} B \beta \Lambda_{0} .
$$

Relembrando que $\Lambda_{1}=2 \Lambda$ e $\Lambda_{0}=\Lambda^{2}$, conlui-se que

$$
P(s) \approx s^{3} I+c_{0} B \beta(s I+\Lambda)^{2} .
$$

Além disso, se $\left\|c_{0} B \beta\right\|_{\infty} \gg\|\Lambda\|_{\infty}$, então

$$
P(s) \approx\left(s I+c_{0} B \beta\right)(s I+\Lambda)^{2} .
$$

Note, considerando a Hipótese 2 e o Teorema do círculo de Gershgorin, que $\beta$ sempre pode ser escolhido tal que os autovalores de $-c_{0} B \beta \in S L E$. Neste caso, o sistema em malha fechada será assintoticamente estável. Além disso, pode-se concluir que

$E(t)=P(s)^{-1} s G+\left(s I+c_{0} B \beta\right)^{-1}\left[c_{0} B \beta-\left(s I+c_{0} B \beta\right)\right] X^{*}$.

Conclui-se então, considerando a Hipótese 1, que $\|E(t)\|$ converge para um conjunto residual que pode ser reduzido aumentando-se $\left\|c_{0} B \beta\right\|_{\infty}$. Além disso, $E(t) \rightarrow 0$ se $G(t), X^{*}(t)$ tenderem a um valor constante. O Teorema a seguir resume o resultado encontrado.

Teorema 1. Considere o problema do controle de rastreamento de trajetória associado ao sistema (1), ao observador de ordem estendida (ESO) com ganhos (28) e à lei de controle (29), na qual $X^{*}$ é a trajetória de referência desejada. Assumindo que as Hipóteses 1 e 2 são satisfeitas, se a matriz simétrica $\Lambda$, a matriz $\beta$ e a constante $c_{0}$ forem escolhidas tal que

- todos os autovalores de $\Lambda$ e $c_{0} B \beta$ pertençam ao SLE; - $\left\|c_{0} \beta\right\|_{\infty} \gg M_{1},\left\|\Lambda_{1} c_{0} B \beta\right\|_{\infty} \gg M_{0},\left\|c_{0} B \beta\right\|_{\infty} \gg$

então:

- $E(t)$ tende a um conjunto residual que pode ser reduzido aumentando-se $\left\|c_{0} B \beta\right\|$;

- $E(t) \rightarrow 0$ se $G(t), X^{*}(t)$ tenderem a um valor constante.

Observação 2. Todo o desenvolvimento matemático que embasou o Teorema 1 foi implementado para um sistema MIMO constituído por três equações de segunda ordem. Esta escolha foi feita somente para facilitar a apresentação da análise, pois todo o desenvolvimento matemático poderia ser facilmente estendido para sistemas com mais de três equações. Além disso, sabe-se que muitos sistemas físicos MIMO são descritos matematicamente por $n$ equações de segunda ordem, o que mostra a generalidade do resultado obtido.

\section{RESULTADOS DE SIMULAÇÃO}

Para os resultados de simulação, foi proposto neste trabalho um exemplo acadêmico, onde foi considerado um sistema não linear que representasse bem as características do controlador. Considere então o sistema descrito por (1) com

$$
\begin{aligned}
& f_{1}(X, W)=-\left[m_{2} \dot{x}_{2}^{2}+m_{3} \sin \left(x_{3}\right)+m_{1} m_{10} x_{1}^{2}+\right. \\
& +m_{1} m_{11} \cos \left(\dot{x}_{2}\right)+m_{1} m_{6} m_{9} \dot{x}_{3}+ \\
& \left.+m_{1} m_{7} m_{9}\left(\sin \left(x_{2}\right)\right)^{2}\right] /\left(m_{1} m_{5} m_{9}-1\right) \text {; } \\
& f_{2}(X, W)=-\left[m_{7}\left(\sin \left(x_{2}\right)\right)^{2}+m_{6} \dot{x}_{3}+m_{2} m_{5} \dot{x}_{2}^{2}+\right. \\
& +m_{3} m_{5} \sin \left(x_{3}\right)+m_{1} m_{5} m_{10} x_{1}^{2}+ \\
& \left.+m_{1} m_{5} m_{11} \cos \left(\dot{x}_{2}\right)\right] /\left(m_{1} m_{5} m_{9}-1\right) \text {; } \\
& f_{3}=-\left[m_{10} x_{1}^{2}+m_{11} \cos \left(\dot{x}_{2}\right)+m_{7} m_{9}\left(\sin \left(x_{2}\right)\right)^{2}+\right. \\
& +m_{6} m_{9} \dot{x}_{3}+m_{2} m_{5} m_{9}{\dot{x_{2}}}^{2}+ \\
& \left.m_{3} m_{5} m_{9} \sin \left(x_{3}\right)\right] /\left(m_{1} m_{5} m_{9}-1\right) \text {; } \\
& B=\left[\begin{array}{ccc}
-m_{4} & m_{1} m_{8} m_{9} & m_{1} m_{12} \\
m_{4} m_{5} & -m_{8} & m_{1} m_{5} m_{12} \\
m_{4} m_{5} m_{9} & m_{8} m_{9} & -m_{12}
\end{array}\right] .
\end{aligned}
$$

onde $m_{1}=1 ; m_{2}=2 ; m_{3}=3 ; m_{4}=1 ; m_{5}=10 ; m_{6}=$ $8 ; m_{7}=9 ; m_{8}=15 ; m_{9}=0,2 ; m_{10}=20 ; m_{11}=$ $15 ; m_{12}=50$. Note que $f_{1}, f_{2}$ e $f_{3}$ são funções não lineares que fazem parte do distúrbio generalizado $G(t)$. Além disso, também são inseridos distúrbios externos $\omega_{1}=\omega_{2}=$ $\omega_{3}=0,5 \cos (5 t)$. Os valores de sintonia dos parâmetros do controlador foram $\gamma=10, \omega_{0}=2500$,

$\beta=\left[\begin{array}{ccc}-4,5455 & 13,6364 & 340,9091 \\ 9,0909 & -2,2727 & 151,5152 \\ 0,3636 & 0,4091 & -2,2727\end{array}\right] \bar{L}=\left[\begin{array}{c}7,49 \times 10^{3} I \\ 1,87 \times 10^{7} I \\ 1,56 \times 10^{10} I\end{array}\right]$

As condições iniciais são $X^{T}(0)=[0,5 ;-0,5 ; 1]$ e a trajetória de referência $X^{* T}(t)=[\cos (2 t) ; 1,5 \cos (2 t) ; 2 \cos (2 t)]$. A Figura 4 mostra o comportamento das saídas $x_{1}, x_{2} \mathrm{e}$ $x_{3}$ do sistema.

Note que as saídas rastreiam as respectivas trajetórias de referência com um erro residual, como pode ser melhor observado na Figura 6, que mostra os erros de rastreamento. A Figura 8 mostra os sinais de controle. Note que os mesmos apresentam grande amplitude nos instantes 


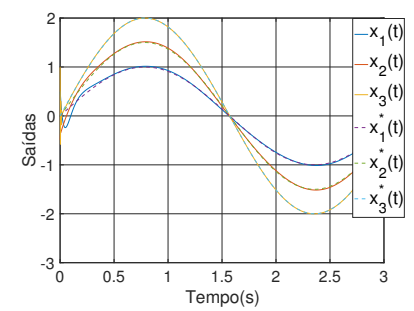

Figura 4. Saídas do sistema

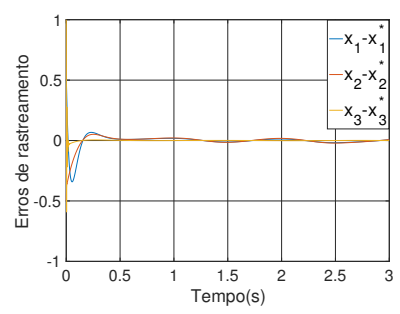

Figura 6. Erros de rastream.

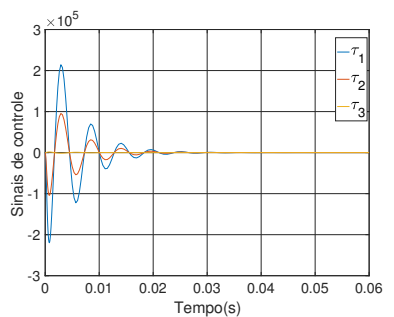

Figura 8. Sinais de controle

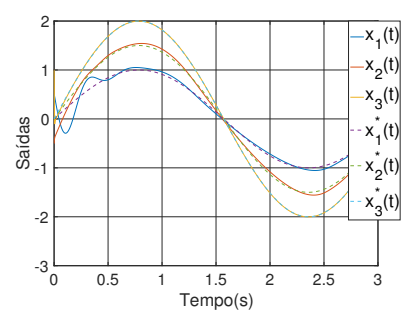

Figura 5. Saídas $(B:=3 B)$

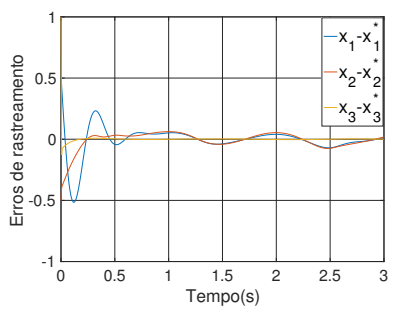

Figura 7. Erros $(B:=3 B)$

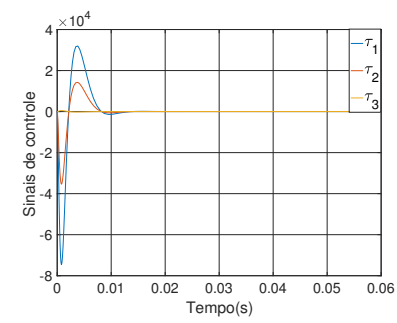

Figura 9. Sinais $(B:=3 B)$

iniciais. Essa é uma característica indesejada, visto que, em um sistema físico, há limites nos sinais gerados pelo atuador. No entanto, esse problema pode ser contornado inserindo-se um filtro de primeira ordem em série com o sistema. Teríamos então um sistema MIMO com três equações de terceira ordem e o controlador poderia ser reprojetado para esta nova condição. Para testar a robustez da estratégia proposta à variação na matriz de ganho de controle $B$, a mesma foi multiplicada por três, mantendose a mesma sintonia do controlador. As Figuras 5, 7 e 8 mostram novamente o desempenho do sistema. Note que não há uma mudança significativa no desempenho. Além disso, note também que o distúrbio externo inserido foi rejeitado em ambos os casos. Esses resultados confirmam a boa robustez da estratégia de controle proposta, tanto quanto à variação na matriz de ganhos de controle, como com relação ao distúrbio externo.

\section{CONCLUSÕES}

Este artigo apresentou um controlador robusto para sistemas incertos com múltiplas entradas e saídas (MIMO). A estratégia utilizou um Estimador de Ordem Reduzida (ESO) para estimar as incertezas, representadas como um distúrbio generalizado, e cancelá-las numa lei de controle de rastreamento. Os resultados de simulação mostraram a eficácia da lei de controle. Como proposta de trabalhos futuros, está a generalização da demonstração para sistemas MIMO de ordem mais elevada e a aplicação da técnica em sistemas físicos reais. Mais especificamente, está em fase de desenvolvimento a aplicação da estratégia proposta no

controle do movimento da asa de um ornitóptero, que é um sistema MIMO, não linear e com forte acoplamento nos torques de entrada.

\section{REFERÊNCIAS}

Chen, C.L.P., G.Wen, Lui, Y., and Liu, Z. (2015). Observer-based adaptive backstepping consensus tracking control for high-order nonlinear semi-strictfeedback multiagent systems. IEEE Transactions on Cybernetics, 46, 1591-1601.

Fossen, T.I. (1994). Guidance and Control of Ocean Vehicles. John Wiley.

Guo, B., Bacha, S., and Alamir, M. (2017). A review on adrc based pmsm control designs. In Industrial Electronics Society, IECON 2017-43rd Annual Conference of the IEEE, 1747-1753. IEEE.

Hsu, L., Oliveira, T.R., and Cunha, J.V.S. (2014). Extremum seeking control via monitoring function and timescaling for plants of arbitrary relative degree. In Proc. International Workshop on Variable Structure Systems. Nantes.

Li, H., Wang, J., Lam, H., Zhou, Q., and Du, H. (2016). Adaptive sliding mode control for interval type-2 fuzzy systems. IEEE Transactions on Systems, Man, and Cybernetics: Systems, 46, 1654-1663.

Mobayen, S. and Tchier, F. (2017). Design of an adaptive chattering avoidance global sliding mode tracker for uncertain non-linear time-varying systems. Transactions of the Institute of Measurement and Control, 39(10), 1547-1558.

Popp, K. and Schiehlen, W. (2010). Ground Vehicle Dynamics. Springer.

Selfridge, J.M. and Tao, G. (2016). Multivariable output feedback mrac for a quadrotor uav. In Proc. American Control Conference, 492-499. Boston (MA).

Sun, L., Li, D., Gao, Z., Yang, Z., and Zhao, S. (2016). Combined feedforward and model-assisted active disturbance rejection control for non-minimum phase system. ISA transactions, 64, 24-33.

Xia, A., Hu, G., Li, Z., Huang, D., and Wang, F. (2018). Self-optimizing pitch control for large scale wind turbine based on adrc. In IOP Conference Series: Materials Science and Engineering, volume 301, 1-8. IOP Publishing.

Xue, W., Bai, W., Yang, S., Song, K., Huang, Y., and Xie, H. (2015). ADRC with adaptive extended state observer and its application to air-fuel ratio control in gasoline engines. IEEE Transactions on Industrial Electronics, 62(9), 5847-5857.

Zachi, A.R.L., Correia, C.A.M., Filho, J.L.A., and Gouvea, A.J. (2019). Robust disturbance rejection controller for systems with uncertain parameters. IET Control Theory and Applications, 13(13), 1995-2007.

Zhang, D., Yao, X., and Wu, Q. (2016). Parameter tuning of modified active disturbance rejection control based on the particle swarm optimization algorithm for highorder system. In IEEE International Conference on Aircraft Utility Systems (AUS), 290-294. Beijing, China.

Zheng, Q., Chen, Z., and Gao, Z. (2009). A practical approach to disturbance decoupling control. Control Engineering Practice, 17, 1016-1025. 